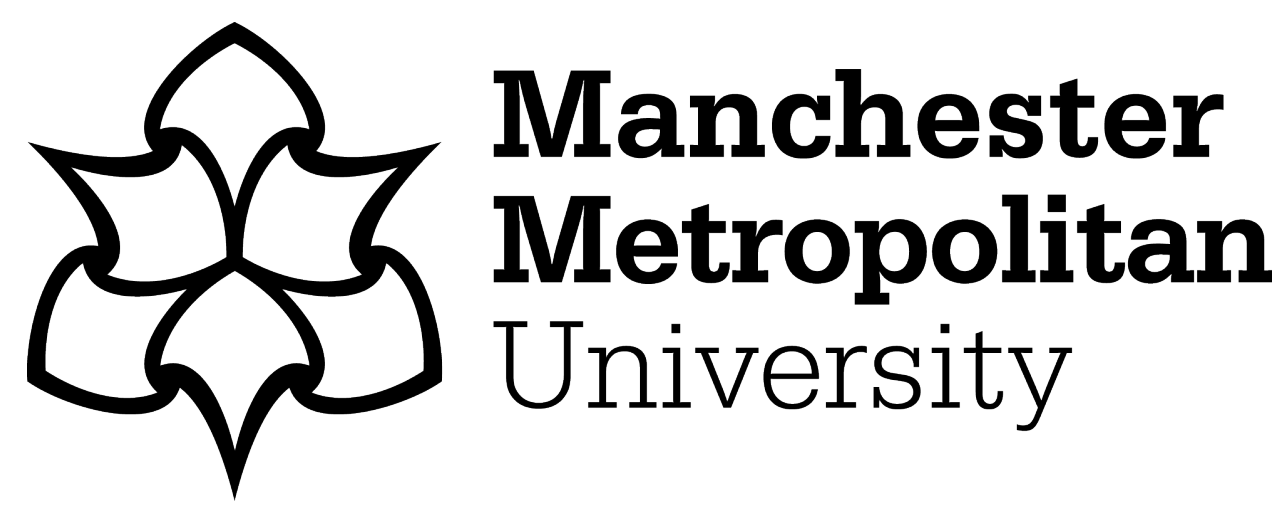

Henry, John ORCID logoORCID: https://orcid.org/0000-0003-3674-8208, Tang, Stephen, Hanneghan, Martin and Carter, Chris (2018) A framework for the integration of serious games and the Internet of Things (IoT). In: 2018 IEEE 6th International Conference on Serious Games and Applications for Health (SeGAH), 16 May 2018 - 18 May 2018, Vienna, Austria.

Downloaded from: https://e-space.mmu.ac.uk/623247/

Version: Accepted Version

Publisher: IEEE

DOI: https://doi.org/10.1109/segah.2018.8401378

Please cite the published version 


\title{
A Framework for the Integration of Serious Games and the Internet of Things (IoT)
}

\author{
John Henry ${ }^{1}$, Stephen Tang ${ }^{2}$, Martin Hanneghan ${ }^{3}$ and Chris Carter ${ }^{4}$ \\ Department of Computer Science, \\ Faculty of Engineering and Technology, \\ Liverpool John Moores University, \\ James Parsons Building, Byrom Street, Liverpool, L3 3AF, UK. \\ Email: j.melthis@2015.ljmu.ac.uk ${ }^{1}$, s.o.tang@1jmu.ac.uk ${ }^{2}$, m.b.hanneghan@ljmu.ac.uk ${ }^{3}$, c.j.carter@1jmu.ac.uk ${ }^{4}$
}

\begin{abstract}
Serious Games and the Internet of Things are research topics of high interest. The integration of these two domains has the potential for innovative new applications. This paper presents a framework for the combination of Serious Games and the Internet of Things. In addition, we present the system architecture for a Smart Serious Game (SSG) developed for measuring student engagement, and define the difference between an event driven game and an SSG. This paper also includes the updated data algorithms for representing student engagement as game points, based on further consideration on our previous publication.
\end{abstract}

Index Terms-Framework; IoT; Serious Games; Smart Serious Games;Data Algorithms;

\section{INTRODUCTION}

The integration of Serious Games and the Internet of Things (IoT) is a research topic that is increasingly attracting attention from the academic community. Literature on the topic is expanding, with investigations on the combination itself [1], frameworks [2], topologies [3], and proposed applications [4], [5]. The term Smart Serious Games (SSGs) was first identified in the book by M. Favorskaya, D. Sharma et al. [1]. This paper will continue to utilise this terminology to describe the technologies.

IoT promises a future of interconnectivity which will allow for more detailed, and extensive analysis of data driven applications and prospects new software solutions that could not have been achieved without IoT. IoT consists of interconnected devices or Things, and data services, that operate in Smart Environments and communicate data with virtual identification and/or personalities [6]. In addition, IoT accounts for an ecosystem which is comprised of middle-ware [7], users and interconnected devices.

Serious Games are computer games built for nonentertainment domains, and have a presence in industries including health, advertisement, training, education, science, research, and others [8]. Serious Games can be entertaining; however, their focus lies on a 'serious' objective. By harnessing the power of entertainment that gaming provides, serious games and gamification have provided a number of research and industrial solutions [9]-[12].

In this paper we present a modular, interconnected framework for SSGs. Literature on such a framework is limited at this time, with research focusing on discovering SSG's potential and defining its presence in the academic field [1], [4], [5]. To address this our framework presents the modular interconnectivity of SSGs by accounting for IoT sensor networks, middleware, and serious games. As the framework is modular, it is also adaptable, for use with other game types such as online games, gamification or edutainment.

In the following section we present the background research surrounding the frameworks of serious games and topologies of IoT. Section III discusses the latest advances in research defining a framework for SSGs and introduces our proposed solution. Section IV details the application of our framework, and the updated data algorithms required for representing student engagement in a serious game. This sections also presents our early data findings regarding validating the proposed framework. In Section V we reflect on the research challenges and suggest future works to be carried out. Finally, Section VI concludes on the key points of this paper.

\section{FRAMEWORKS AND TOPOLOGIES}

In a truly interconnected IoT environment, Serious Games could harvest and analyse data from players physical worlds and present it to users, generating meaningful gameplay representations. The combination of Serious Games and IoT has recently been termed as Smart Serious Games (SSGs) [1]. SSGs have been defined as the integration of smart technologies, including devices and services, and the principles of Serious Games [1]. This literature details the combination of the advantages of both technologies and its future utilisations including; analytics for cooperations, a tool for solving serious problems and others.

As this term is new, literature on SSGs is limited, with some research projects beginning to include the term as future works [13], [14]. It is very important to continue research into SSGs as IoT brings better data acquisition that creates quantitative results and a non-intrusive experience.

Currently, there is a limited number of modular frameworks for the combination of Serious Games and the Internet of Things. Literature is continuously emerging; however most are of a preliminary nature, or are service specific [2], or offer insights towards a framework for SSGs [15]. 


\section{A. Serious Games Frameworks}

There is a vast amount of literature surrounding serious game methodologies, and frameworks, including surveys of such publications. This section provides the identified research pieces that illustrate some form of modular framework for serious games.

S. Tang and M. Hanneghan identified a model-driven framework for Serious Games. Their paper encourages the development of serious games for educational purposes by multi discipline domains. Their framework is modular and therefore encourages adaptation for service specific applications [8]. The modular approach will be reused for this framework to allow future works to adapt it to meets a project's needs.

S. Arnab et.al., introduced a framework for Serious Games for pedagogical use [16]. Their framework focuses on coherently merging the attributes of pedagogy and games in order to produce better educational games. S. Arnab et. al., outline a detailed and effective framework for use in such games, elements of which can be applied to event-driven games in an educational setting, such as this research's. However, this research project furthers any form of Serious Games framework by merging IoT and outlining the modular interconnection between the technologies.

K. Killi et.al. presented a framework for achieving flow in educational games [17]. Their paper focuses on linking educational theory with game design and presents a valid contribution to maintaining engagement in educational games. Their research is of a service specific nature and provides additional points for consideration when defining a framework for SSG's.

B. Cowley et.al. published a novel approach to serious game design by introducing smaller game elements into a framework instead of constructing a service specific one [18]. This approach leads to the creation of modular framework where development can start at any stage based on the principle developed. This modular approach coexists in our solution; however, we focus on producing a modular framework for the integration of IoT and Serious Games, that can be utilised to accelerate the production of such applications and further the surrounding academic field.

Research into serious games for obesity by M. Hassan et.al. produced a framework capable of obtaining real time sensor information from Body Sensor Networks (BSNs) that feed into the game and suggest improvements directly to the players regarding exercise and nutrition. [15]. This real time approach has been adopted by elements of our framework, as discussed below. M. Hassan et.al, successfully outline the technologies required for a game that was labelled as pervasive but can be considered for IoT. We extend this type of research by defining an application-neutral topology with a modular outline, that will aid researchers to develop service specific Serious Games or Games that embed IoT.

Due to the physical and networking nature of IoT, topologies can be more useful than frameworks for IoT based applications. We have proposed a topology for the application area of this research, detailed in the Section IV, and have reviewed current game and IoT topologies to suggest those best for the development of SSGs [3]. A plethora of research exists on service specific topologies for IoT. In previous research we have identified those that relate closest to Serious Games [3].

\section{Smart Serious Games Framework}

There is a distinct lack of frameworks for the integration of Serious Games and IoT. Some research is beginning to prevail such as H Kim's paper [2], which discusses the integration of IoT and games with games as a service. Through his paper, a number of circuit diagrams arise as well as blueprint for the interconnection of mobile clients and server. As his research is service specific and of a preliminary nature, the paper falls short of producing a re-usable, modular framework.

Existing research into SSGs outline bespoke frameworks [2], applications [4], [5] or extensively define the academic spectrum [1]. The previous section outlined the benefits of using modular frameworks for Serious Games, highlighting the vast potential for applications and the ease of adjustment for service specific solutions. Therefore, this paper continues with the proposition of a modular framework for the representation of combining Serious Games and IoT.

Producing a modular framework for the integration of Serious Games and IoT requires a neutral perspective, in which fellow researchers may swap or adjust the framework to suit their research's needs. To achieve this, our framework clearly identifies the technological boundaries of Serious Games and IoT and demonstrates the interconnecting technologies in a top-down hierarchy. We accept that for service specific applications different hierarchy styles may be chosen, however a top down style is the easiest to comprehend for this illustration.

\section{A. Requirements}

In architecting the framework, we determined the key requirements needed for producing a framework for Serious Games and IoT, based on the aforementioned research. By meeting the following requirements, frameworks can ensure they provide a basis for a vast variety of applications within their domain.

1) Scalability: This requirement echoes the requirement we set in our previous research, when defining topologies for Serious Games and IoT [3]. In online games, whether they be serious or not, the amount of players can increase drastically pending on popularity spikes. We often see commercial games struggle to accommodate for players at lunch as they incorrectly allocate resources at the server end. A relatively recent example of such as scenario was the lunch of Grand Theft Auto $\mathrm{V}$ which saw players struggle to connect online due to the volume of requests. By incorporating IoT, new challenges arise. New locations could be added at any point for games that are location based. This would equate to new nodes or new sensor clusters that need to be connected to the same framework. New extrinsic sensor networks could also be added using Application Programming Interfaces (API). Therefore, scalability of a framework is essential for Smart Serious Games. 
2) Topology neutral: At the end of the previous section we discuss topologies. As we are merging IoT with Serious Games, topologies form a core element of the integration between the two. It is the topology that will define the networking requirements of the framework. A challenge of developing frameworks for Smart Serious Games is avoiding defining one, based off a single topology, as this would limit the scope and risk the framework becoming service specific.

3) Application neutral: By application neutral, we describe a framework that is not service specific. Application neutral frameworks present greater impact. The background research [2], provides an example for a lack of scope coming with a proposed framework, as there are specifics embedded in, that tailor to a single application.

\section{B. Proposed Solution}

Based on our findings we propose the modular framework seen in Fig.1. The framework considers the data flow to begin from the top and end at the bottom. This flow is not the only form of data flow that can be had. Data will flow from the application layer through to the middleware layer. From there data will flow back down, updating the game state. This will form a data flow loop which allows game progression based on user input. An example of such loop would be purchasing an item in game. The game triggers the data request, if this request meets the correct conditions (user balance) then the request will be granted, and the game will be updated to reflect the change in inventory.

Our framework encompasses of five layers; sensing, networking, middleware, data processing and application. Below we break down each layer in detail.

1) Sensing layer: Within the sensing layer we include two modules; intrinsic and extrinsic sensor networks. Intrinsic sensor networks describe physical networks that have been developed or established for an application. These networks can be intrusive (human body sensor network, RFID) or non-intrusive (Bluetooth, Wi-Fi). Extrinsic sensor networks detail pre-existing networks that the users of an application may not come in direct contact with, whether intrusively or not. Such networks can be found in APIs such as traffic, weather and others, where large based sensor networks feed in environmental data for various purposes. For our application, we incorporate extrinsic sensor networks to correlate student behaviour with traffic and weather. Other applications can tailor these modules to adapt to their requirements.

2) Networking layer: The networking layer houses all the essential technologies to allow data communication of sensor networks to middleware. The communication technology module provides basic networking and data communication over a wireless or wired network, including WWAN, WPAN, WLAN [19]. For our application cellular networks such as 3G, 4G and others, can also be included, as the game requires internet connectivity to operate. The communication protocols module pin points the underlying protocols that are utilised within the networks. Bluetooth Low Energy (BLE) enabled application could benefit from specifying the Generic Application Profile

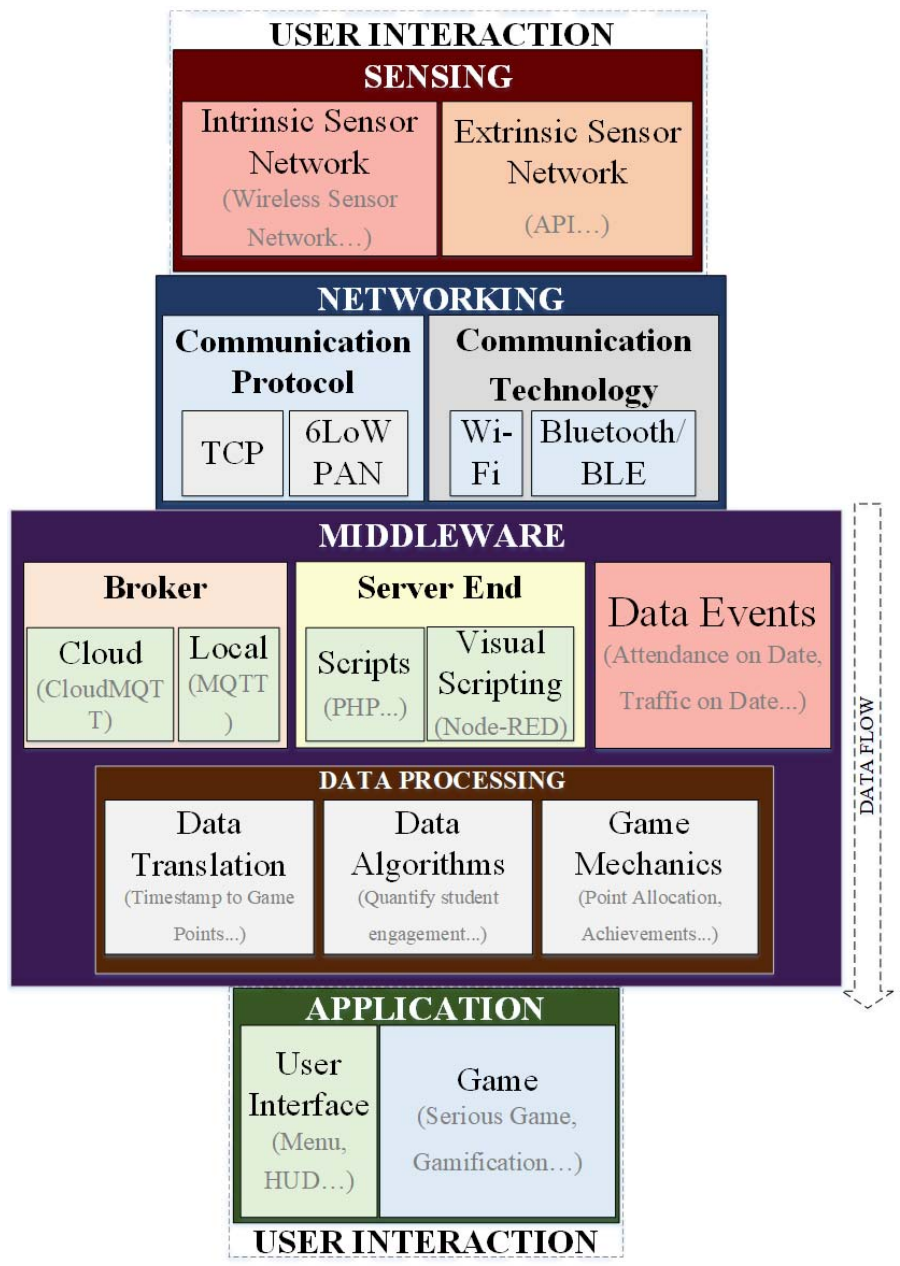

Fig. 1. Smart Serious Games Framework

(GATT) protocol within this module. An argument can be made for the placement of this module in our figure, as the application would need to access the networking layer to communicate with the middleware. Though this is true, for readability we focus on one directional data flow. Aside of this, networking is expected to be essential for an online game, and therefore it's placement can be presumed.

3) Middleware layer: The middleware layer forms the bridge between all layers, and for some data streams serves as an end point. In the middleware we find the broker module, which in turns accommodates for local or cloud-based brokers. Four our application we have utilised a cloud-based broker (CloudMQTT) that handles messages sent from an direct sensor network to the server. We also place the server end module here, in which we specify web-hosted PHP scripts and local scripts executed through Node-RED. These technologies can vary based on the application needs, for example, a hosted web-app and database alone may suffice for other applications. The final module outlined in this layer is Data Events. A Smart Serious Game will have some form of data events disregarding its application. Data events include triggering game notifications based on time, allocating points based on a 
players physical location in a given moment, within the direct sensor network, and others. The events themselves can greatly vary based on the application being built, however a game that does not require data events may not require the integration of IoT at all.

4) Data processing layer: The data processing module is housed by the middleware layer, and concerns a number of processing steps for handling raw sensory data [20], and other data derived from gameplay, including the construction of data storage centres, search engines, smart decisions and data mining approaches. Three modules are included in this module; data translation, data algorithms and game mechanics. All three modules play a pivotal role for SSG based applications. The sensory data must be translated into a meaningful resource that can be utilised in game. For example, a Bluetooth network that detects your presence at a specific date and time can be translated into game points, rewarding the user for being at a physical space within a set time and date. Data algorithms can then be used to summarise or aggregate these points for use within a game, hence we specify the data algorithm within this module too. It is the data algorithm module that could be removed for service specific applications, where sensory data play a singular role within a game. Finally, we specify game mechanics in this module. It is the game mechanics that directly interact with data translation and trigger a reaction within a game, such as levelling a character up, unlocking an achievement and others.

5) Application layer: The application layer provides an interface for user interaction [21], [22], as specified in the user interface module, and defines the game itself. The game does not have to be a serious game. Gamification and games can both benefit from this framework, as it is the integration with IoT that holds value. An argument can be made for the removal of the user interface module, as it is possible to create games that merge with IoT with no interface at all. An example of this could be a game that is played with buzzers. The buzzers instruct play and react to a player's physical location. The modular approach allows future application to remove the user interface module without interfering with the stated layers.

\section{SEA: Student Engagement Application}

Student engagement is conventionally measured through questionnaires [23]-[27]. This paper investigates the effectiveness of measuring student engagement by gamifying the process through a serious game. In SEA, the aim is to keep your avatar happy by achieving high scores. Fig.2 showcases the main screens players interact with. SEA calculates engagement on a weekly basis (every Monday), allowing students to complete their questionnaire over the weekend. We utilise a data algorithm to put a value to a student's engagement level, and award game currency on a 1:1 basis, where one point of engagement is equal to one value of currency. Players can purchase new skins for their avatars and unlock achievements as they progress. The combination of the aforementioned game mechanics provides a well-rounded experience that immerses players. In addition, we designed a global leader board to

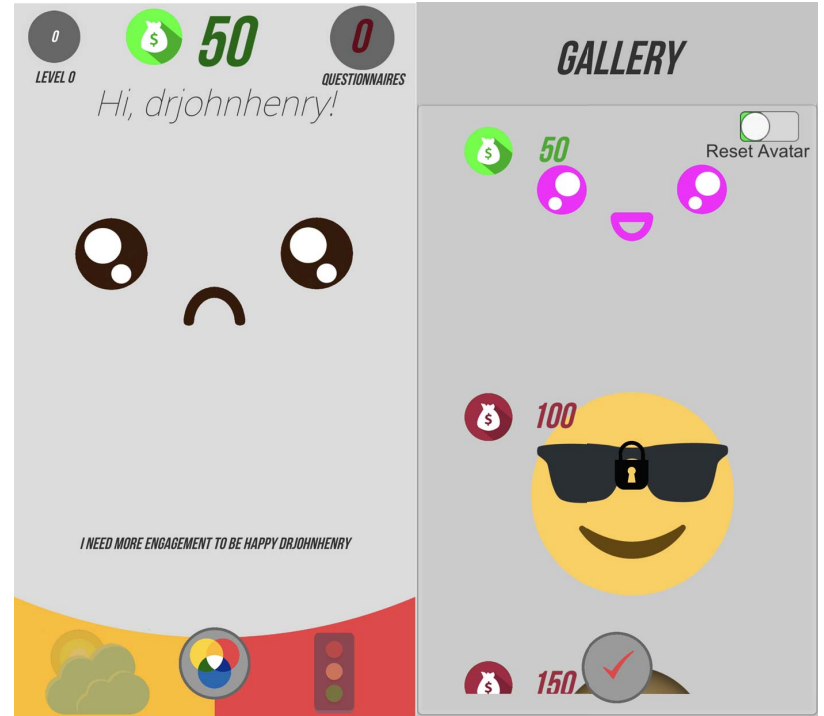

Fig. 2. SEA: Student Engagement Application.

add an element of competitiveness. In the Game Architecture section, we discuss the extended involvement of IoT in our game, aside of the wireless sensor network that monitors attendance and punctuality.

Our previous publication [28], suggested a data algorithm for measuring student engagement and defined the game points to be allocated. We have adjusted the data algorithm to reduce the strain on lecturers and students alike. Initially, we proposed a student completes daily questionnaire that sources their subjective engagement, whilst the appropriate lecturer completes the same questionnaire, obtaining the perceived level of engagement for the related student. We theorised that the mean score would produce a form of validation. Psychological research in student engagement obtains scores solely from the student and at far lesser intervals, therefore this approach was abandoned. Engagement $(E n)$ is the outcome of Score $(S)$ divided by the highest possible score $(\mathrm{He})$ each student could achieve. As the obtained value is a percentage, we multiply by one hundred. This algorithm presents a limitation for use in games. Games scores or points are rarely represented as decimals, therefore we apply the round-ceiling algorithm to the score produced, ensuring we always obtain a whole number, in favour of the student.

$$
S=\left[\frac{((C a+C p)+W t)}{2}\right]
$$

To understand the algorithm, we must define Score and Highest engagement. Score is the sum of calculated attendance $(C a)$, calculated punctuality $(C p)$, and questionnaire feedback, divided by two. We divide by two to obtain the mean value of IoT generated score and electronic questionnaires.

$$
H e=\left[\frac{(A p * T c)(P p * T c)+(H Q S)}{2}\right]
$$


Highest engagement is a score relative to a student as it considers the amount of weekly timetabled classes. In detail, $H e$ comprises of $A p, P p, T c$, and $H Q S$.

- Ap: A static game point allocated by the game developer for attending a class.

- Pp: A static game point allocated by the game developer for punctuality with a class.

- Tc: The amount of weekly timetabled classes for a student.

- HQS: The highest measure from the questionnaires. More information on questionnaire scoring can be found in our previous publication [28].

Therefore, the highest possible engagement score is the best attendance score added to the best punctuality score for week, which the sum of is added to the highest possible measure of the questionnaire scores. Previously we divided the score algorithm by two to find the mean measure of questionnaire and IoT measurements, for consistency to that approach we divide the total sum by two.

$$
E n=\left[\left(\left(\frac{S}{H e}\right) 100\right) \text { Round }- \text { Ceiling }\right]
$$

By altering the data algorithm for representing student engagement in a game, we consider the persona of student that prefers distance learning, through virtual learning environments. Previously, most game coins were achieved through attendance and punctuality. We simplified the algorithm to calculate the mean value of attendance $C a$ plus punctuality $C p$, added to the total score achieved from the questionnaire $W t$. To elaborate, where a student receives five points for attendance and an additional five for punctuality, in a week of five classes, they could achieve fifty points. If the same student states he is fully engaged through the questionnaire he would accumulate an additional 40 points. However, the game coins he would receive for the week would amount to 45 . In addition, by calculating the mean, we follow university procedure, which states a student must attend at least one class a week. Mathematically, a student that attends one class but selfreflects their selves as being engaged could achieve a score of 25 game coins, and progress well through the game. Previous literature [25] states academic performance as the outcome of engagement. Therefore, at the end of the experiment period, to validate, we will calculate academic performance with the measure we obtained. Further information on the experiment process can be found in the Experiments section below.

\section{A. Game Architecture}

We developed SEA utilising the following system architecture, seen in Fig.3.

In detail, for our application we considered buildings as location variances in oppose to rooms within the buildings. If we were to consider each room that a lecture or lab took place in, the edges of the network would have grown exponentially, meaning hundreds of Raspberry Pi would have been required to complete such coverage. To solve this, we utilised the $\mathrm{Pi}$ as a mobile node. Each lecturer involved in experiments will receive a $\mathrm{Pi}$ that they take to their practical and lecturing sessions. The architecture we present is fully scalable, and therefore would accommodate for static nodes, however this is not necessary for the scope of this research. For our system architecture, the lecturer is provided with a portal that communicates with the cloud broker and the local based visual scripting tool, to send a message to the appropriate $\mathrm{Pi}$ that set's the building's location. This process is bidirectional, as once the location is set successfully, the appropriate lecturer will receive a confirmation email. The aforementioned detail the choice of two locations (A, B) and four Pi, other network nodes can be utilised with the same result. We opted for the Raspberry Pi 3 as it offers on-board Wi-Fi and Bluetooth.

In each location, the architecture specifies numerous smartphones connecting to a $\mathrm{Pi}$. This process establishes the presence of a said participant in a given room. A powered-on $\mathrm{Pi}$ will scan it's environment for a list of Bluetooth Addresses that it retrieves from the database. These Bluetooth addresses form the identification of a participant for the system. If a relevant Bluetooth Address is found in the Pi's environment, the name of the building along with the Bluetooth Address is sent across through the cloud broker to the local visual scripting tool. The tool then triggers a hosted script that validates the presence against a said participants timetable. If the time and location of the data received are within the confines of a lecture or practical sessions, the data will be recorded in a database and a second hosted script will trigger a notification through the Batch $\mathrm{API}^{1}$, to the participants phone, acknowledging their presence. Batch gratefully decided to support this research project for this feature. If the data is outside the time scope of a timetabled lecture or practical session, the data process ends.

The system architecture states the inclusion of traffic and weather data. For our system, traffic is obtained an hour in advance of a class, as long as a player has specified they travel to campus by car. This data is then stored in the database for further analysis with a player's behavioural pattern. We utilise the HERE API to obtain traffic data. Weather data is obtained on a daily basis, from Monday to Friday. The weather API used is the met office.

Developing a game that is event driven is hardly a smart serious game. We define our game as an SSG due to these key factors:

1) Data from the real world (traffic, weather) directly feed into the game and are presented to the end user.

2) Heterogeneous devices are intercommunicating for the purpose of the game.

3) The game utilises data sourced from direct sensor networks to affect gameplay.

We utilise API data to correlate 'bad' behavioural habits that are relational to student engagement. In detail, for each time a player is late or absent to a class the game will get a list of the weather and traffic conditions for that day and time. From there, the game detects which condition re-occurred

\footnotetext{
${ }^{1}$ www.batch.com
} 


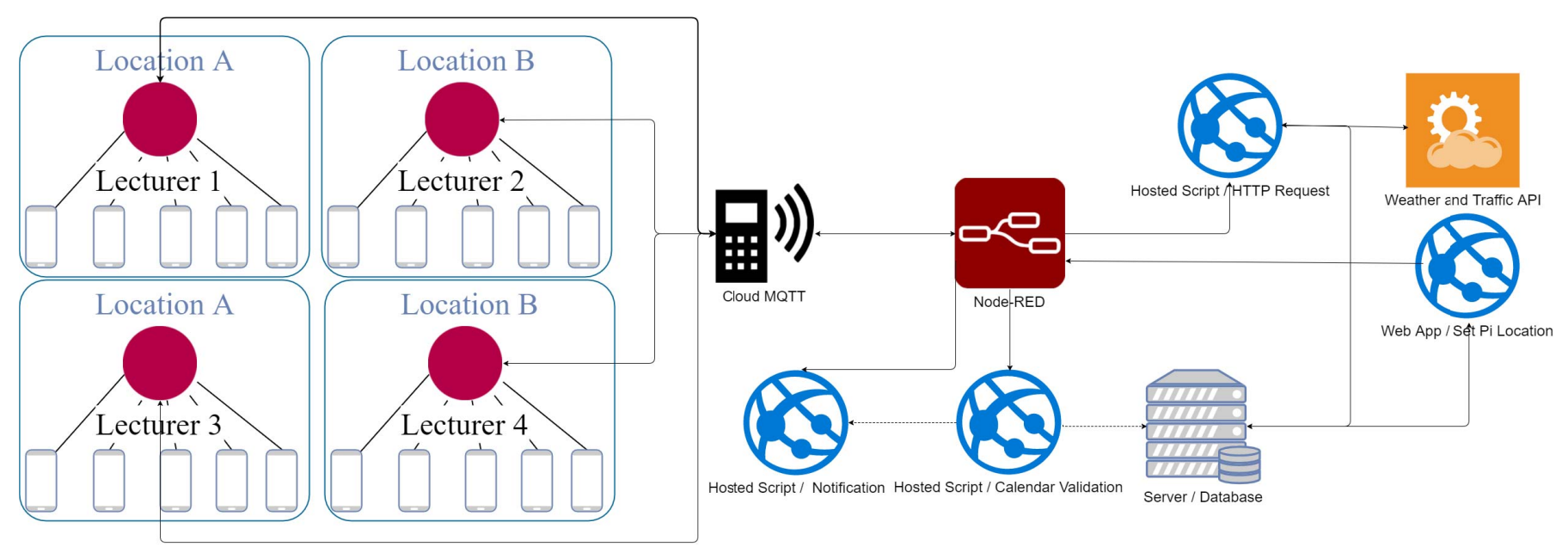

Fig. 3. System architecture for developed SSG.

most commonly and present this to the user in-game. For example, where a said player has been absent for $x$ number of classes in a week, the program will uncover the most common condition, such as Heavy Rain, and inform the participant. The information changes on a weekly basis. By presenting this type of information to the end user, it is possible they will uncover patterns regarding their behaviour, that they may choose to change. We utilise traffic and weather APIs due to cost limitations. A host of alternative environmental data exists, such as public transportation, air quality and others. We recommend utilising as much API data in SSGs to enrich the experience and the information provided to the player, where possible.

\section{B. Experiments}

To examine the effectiveness of our framework, we developed a multi-system game that merges IoT with Serious Games. The serious game quantifies behavioural student engagement and utilises questionnaires to measure cognitive and emotional engagement as practised in psychological research [28]. Research has been conducted into the measuring engagement of games through a questionnaire by J. Brockmyer et.al. [27]. They uncovered that engagement in game is quantifiable. This supports quantifying student engagement. V. Riemer and C. Schrader researched the behavioural measurements for learning through serious games, to bridge the gap in research surrounding the area [29]. Their research found a difference in attitudes based on the serious game type, for example simulation, and the gender that played the game, however, positive attitude was generated through play for learning.

Subsequently, we are undertaking experiments that aim to prove the validity of our proposed framework, by utilising control groups. We have managed to recruit undergraduate students from all three years of degree study. The total amount of participants $(n=22)$ were randomly divided into two groups, named Group A and Group B. In Group A, participants were asked to complete a questionnaire that measures engagement with their programme of study. The questionnaire was devel- oped based on the aforementioned research but adjusted to ensure that a positive response gave a higher score. To increase the possible response rate from questionnaires we followed the recommendations detailed in a systematic review into electronic and postal questionnaires conducted by P. Edwards et al. [30], and ensured our electronic questionnaire comprised of a white background, textual representation for responses and had a set deadline for completion. The questionnaire was adjusted to account for Group B, where participants played our Serious Game and completed the same questionnaire in-game. By ensuring a positive result gave a higher value, we could input the questionnaire responses into the aforementioned data algorithm for engagement.

By utilising control groups we aim to correlate the measurements of engagement (conventional vs SSG), and in turn, validate the proposed framework for the integration of Serious Games and IoT. In addition, we aim to identify any patterns of improvement in student engagement by examining the standard deviation and the root mean square error of the generated data. At the end of experiments, we will collect qualitative data from participants that will be utilised to perform data triangulation on all three points of data.

Experiments will continue to run until the end of the semester, when data analysis will be performed, however we expect that the framework will be fully validated when the experiment is concluded. Initial data showcases a better measurement in participants that are playing the game in comparison to those that are completing electronic questionnaires. Preliminary analysis on the data showcased no issue with intervention. Both groups of participants receive constant reminders, through email and notifications respectively. As emails are available on student phones it is hard to argue for the minor difference in medium. The set of students that need to complete the electronic questionnaires have also received human intervention. This explains the hike in participation in Week 3. Group B also received human intervention with outstanding questionnaires in Week 3, balancing the effect on both data sets. 


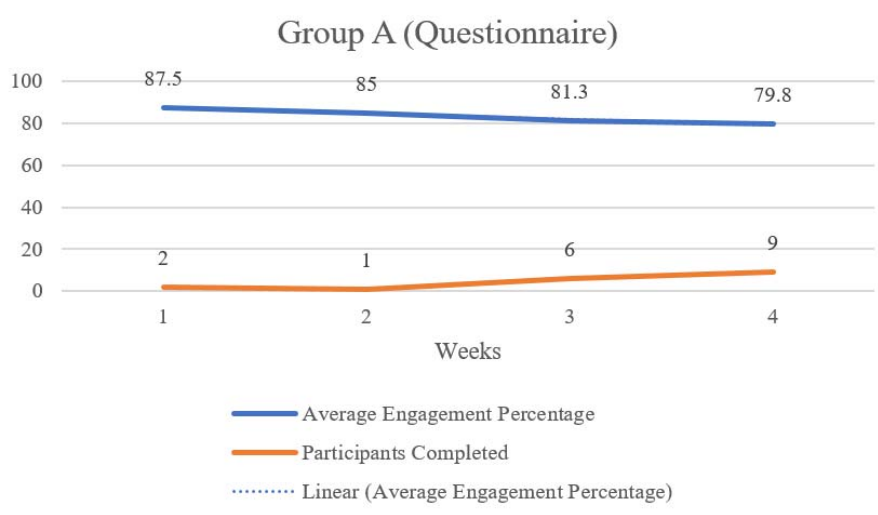

Group B (Questionnaire and SSG)

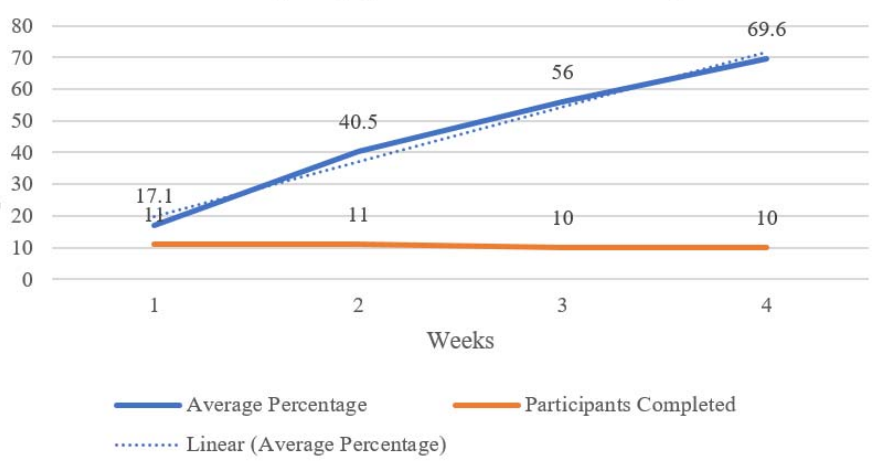

Fig. 4. Current data.

At the time of writing, Group A has a response rate of $40.90 \%$ whereas Group B has a total of $95.45 \%$. Further analysis identified some in Group B to have played the game but not complete their questionnaire, producing a total $33.33 \%$ of erroneous data. We expect to see no improvement for the stated weeks in Group A, however, we expect Week 3 and 4 in Group B to significantly improve as more students launch the, and in turn provide an automated response to their engagement percentage.

Our research project set out two hypotheses; A modular framework can be defined for the integration of Serious Games and IoT, and we can measure student engagement through SSGs. The aforementioned analysis supports both.

\section{Research Challenges and Future Directions}

In this paper we have presented a framework for the developing SSGs. Based on this framework we have developed a game that utilises IoT [29], data algorithms [28] and game engine technology to measure student engagement, by quantifying behavioural engagement and embedding cognitive and emotional engagement as game points through gameembedded questionnaires. At the time of writing, experiments are being conducted with university students from Liverpool John Moores University to determine the effectiveness of measuring student engagement through an SSG.

There is a challenge in measuring the engagement of students that choose to learn from distance. Virtual Learning Environments, and Open Courses present a new format of learning, where behavioural engagement cannot be measured through attendance nor punctuality. This challenge can be overcome by accounting for the background of the student and the level of the material being covered. This can be fed into the system through API sources or learning analytics systems in conjunction with IoT devices. Additional factors that can be considered include class size, which can affect students with social anxiety. We propose such changes as future works to improve the measure of engagement and accommodate for a larger persona of student.

Finally, measuring Group A, without a monetary incentive is proving challenging. $\mathrm{K}$ Ashton [31] stated in his review of electronic questionnaires that response rate is a disadvantage of electronic questionnaires, even though other research [32], [33] identifies better response rates in electronic questionnaires in comparison to postal. The addition of a SSG, is indicating better promise for the reduction of response rates and in turn better measurements of engagement.

\section{CONCLUSION}

This paper introduces a framework for merging Serious Games with the Internet of Things. Our framework takes a modular and interconnected approach while maintaining application neutrality. Furthermore, we present a set of requirements for creating a framework for SSGs. In addition, we presented the bespoke system architecture for a SSG, developed for measuring student engagement. At the time of writing, experiments are being undertaken and early results present promise to the effectiveness of our framework and measure. The SSG, however, successfully operates with user input from appropriate lecturers and students, and data being obtained from API data and sourced from the direct sensor network, therefore getting a SSG to operate through the defined framework has proved successful. In addition, the initial results from the experiments being undertaken present an accurate measure of engagement, with results from the experiments being carried out will be disseminated accordingly.

\section{REFERENCES}

[1] D. Sharma, J. C. Lakhmi, M. Favorskaya, and R. J. Howlett, Fusion of Smart, Multimedia and Computer Gaming Technologies. Springer International Publishing, 1 ed., 2015.

[2] H.-y. Kim, "A design and implementation of a framework for games in IoT," The Journal of Supercomputing, feb 2017.

[3] J. Melthis, S. Tang, P. Yang, M. Hanneghan, and C. Carter, "Topologies for combining the internet of things and serious games," Journal of Intelligent and Fuzzy Systems, vol. 31, no. 5, pp. 2685-2696, 2016.

[4] E. I. Konstantinidis, "The interplay between IoT and serious games towards personalised healthcare," 9th International Conference on Virtual Worlds and Games for Serious Applications, pp. 249-252, 2017.

[5] C. Garcia-Garcia, F. Terroso-Saenz, F. Gonzalez-Burgos, and A. F. Skarmeta, "Integration of serious games and IoT data management platforms to motivate behavioural change for energy efficient lifestyles," GIoTS 2017 - Global Internet of Things Summit, Proceedings, 2017.

[6] L. Atzori, A. Iera, and G. Morabito, "The Internet of Things: A survey," Computer Networks, vol. 54, no. 15, pp. 2787-2805, 2010.

[7] Z. Khalid, N. Fisal, and M. Rozaini, "A Survey of Middleware for Sensor and Network Virtualization," Sensors, vol. 14, no. 12, pp. 24046-24097, 2014.

[8] S. Tang, M. Hanneghan, and C. Carter, "A Platform Independent Game Technology Model for Model Driven Serious Games Development.," Electronic Journal of e-Learning, vol. 11, pp. 61-79, jan 2013. 
[9] I. Blohm and J. M. Leimeister, "Gamification: Design of IT-based enhancing services for motivational support and behavioral change," Business and Information Systems Engineering, vol. 5, no. 4, pp. 275278, 2013.

[10] J. W. Burke, M. D. J. McNeill, D. K. Charles, P. J. Morrow, J. H. Crosbie, and S. M. McDonough, "Optimising engagement for stroke rehabilitation using serious games," Visual Computer, vol. 25, no. 12, pp. 1085-1099, 2009.

[11] C. Girard, J. Ecalle, and a. Magnan, "Serious games as new educational tools: How effective are they? A meta-analysis of recent studies," Journal of Computer Assisted Learning, vol. 29, no. 3, pp. 207-219, 2013.

[12] C. Aldrich, "The complete guide to simulations and serious games :how the most valuable content will be created in the age beyond Gutenberg to Google," Pfeiffer essential resources for training and HR professionals, vol. 1, p. 533, 2009.

[13] E. Lotfi and B. Mohammed, "Players Performances Analysis based on Educational Data Mining Case of Study : Interactive Waste Sorting Serious Game," International Journal of Computer Applications, vol. 108, no. 11, pp. 13-18, 2014.

[14] L. Elaachak, A. Belahbibe, and M. Bouhorma, "Towards a System of Guidance, Assistance and Learning Analytics Based on Multi Agent System Applied on Serious Games," International Journal of Electrical and Computer Engineering (IJECE), vol. 5, no. 2, pp. 344-354, 2015.

[15] M. M. Hassan, M. S. Hossain, A. Alamri, M. A. Hossain, M. AlQurishi, Y. Aldukhayyil, and D. T. Ahmed, "A cloud-based serious games framework for obesity," Proceedings of the 1st ACM multimedia international workshop on Cloud-based multimedia applications and services for e-health - CMBAS-EH '12, p. 15, 2012.

[16] S. Arnab, T. Lim, M. B. Carvalho, F. Bellotti, S. De Freitas, S. Louchart, N. Suttie, R. Berta, and A. De Gloria, "Mapping learning and game mechanics for serious games analysis," British Journal of Educational Technology, 2015.

[17] K. Kiili, T. Lainema, S. de Freitas, and S. Arnab, "Flow framework for analyzing the quality of educational games," Entertainment Computing, vol. 5, pp. 367-377, dec 2014.

[18] B. Cowley, J. L. Moutinho, C. Bateman, and A. Oliveira, "Learning principles and interaction design for 'Green My Place': A massively multiplayer serious game," Entertainment Computing, vol. 2, no. 2, pp. 103-113, 2011.

[19] D. Guinard, S. Karnouskos, V. Trifa, B. Dober, P. Spiess, and D. Savio, "Interacting with the SOA - based Internet of Things : Discovery, Query , Selection , and On - Demand Provisioning of Web Services," IEEE Transactions on Services Computing, vol. 3, no. 3, pp. 223-235, 2010.

[20] B. Y. Xu, L. D. Xu, and E. H. M. Cai, Ubiquitous data accessing method in IoT-based information system for emergency medical services, vol. 10. Issue: 2, 2014.

[21] S. Fang, L. Xu, H. Pei, Y. Liu, Z. Liu, Y. Zhu, J. Yan, and H. Zhang, “An Integrated Approach to Snowmelt Flood Forecasting in Water Resource Management," Industrial Informatics, IEEE Transactions on, vol. 10, pp. $548-558$, feb 2013.

[22] J. Gubbi, R. Buyya, S. Marusic, and M. Palaniswami, "Internet of Things (IoT): A vision, architectural elements, and future directions," Future Generation Computer Systems, vol. 29, no. 7, pp. 1645-1660, 2013.

[23] J. J. Appleton, S. L. Christenson, D. Kim, and A. L. Reschly, "Measuring cognitive and psychological engagement: Validation of the Student Engagement Instrument," Journal of School Psychology, vol. 44, no. 5, pp. 427-445, 2006.

[24] B. D. Coller and D. J. Shernoff, "Video Game-Based Education in Mechanical Engineering: A Look at Student Engagement," International Journal Of Engineering Education, vol. 25, no. 2, pp. 308-317, 2009.

[25] M. M. Handlesman, W. Briggs, N. Sullivan, and A. Towler, "A measure of college student course engagement.," The Journal of Educational Research, vol. 98, no. 3, pp. 184-192, 2005.

[26] E. A. Skinner and M. J. Belmont, "Motivation in the classroom: Reciprocal effects of teacher behavior and student engagement across the school year.," Journal of Educational Psychology, vol. 85, no. 4, pp. 571-581, 1993.

[27] J. H. Brockmyer, C. M. Fox, K. A. Curtiss, E. Mcbroom, K. M. Burkhart, and J. N. Pidruzny, "Journal of Experimental Social Psychology The development of the Game Engagement Questionnaire : A measure of engagement in video game-playing," Journal of Experimental Social Psychology, vol. 45, no. 4, pp. 624-634, 2009.
[28] J. Henry, S. Tang, M. Hannenghan, and C. Carter, "A Measure of Student Engagement for Serious Games and IoT," in Edutainment 2017, 2017.

[29] V. Riemer and C. Schrader, "Learning with quizzes, simulations, and adventures: Students' attitudes, perceptions and intentions to learn with different types of serious games," Computers and Education, vol. 88, pp. 160-168, oct 2015.

[30] P. J. Edwards, I. Roberts, M. J. Clarke, C. DiGuiseppi, R. Wentz, I. Kwan, R. Cooper, L. M. Felix, and S. Pratap, "Methods to increase response to postal and electronic questionnaires," 2009.

[31] K. B. Wright, "Researching Internet-Based Populations: Advantages and Disadvantages of Online Survey Research, Online Questionnaire Authoring Software Packages, and Web Survey Services," Journal of Computer-Mediated Communication, vol. 10, no. 3, pp. 00-00, 2006.

[32] D. Andrews, B. Nonnecke, and J. Preece, "Electronic Survey Methodology: A Case Study in Reaching Hard-to-Involve Internet Users," International Journal of Human-Computer Interaction, vol. 16, no. 2, pp. 185-210, 2003.

[33] H. E. Drummond, S. Ghosh, A. Ferguson, D. Brackenridge, and B. Tiplady, "Electronic quality of life questionnaires: a comparison of pen-based electronic questionnaires with conventional paper in a gastrointestinal study," Quality of Life Research, vol. 4, no. 1, pp. 21-26, 1995. 